\title{
FAHR'S DISEASE: A RARE NEURODEGENERATIVE DISORDER IN CHILDREN
}

\author{
RAHMAN AKMM ${ }^{1}$, BEGUM RS $^{2}$, HOSSAIN MZ $^{3}$, ALI MR $^{4}$, RAHMAN M ${ }^{5}$
}

\begin{abstract}
:
A case of Fahr's disease in reported in a 2 year 9 month old male child presented with developmental delay and seizure. The CT scan of brain showed multiple symmetric calcifications in the basal ganglia, thalamus, and subcortical white matter of cerebral and cerebellar areas. No underlying cause for the bilateral calcification was found. This rare case of Fahr's disease in a child, which has never been reported in Bangladeshi literature, has been brought out to highlight this unusual condition.
\end{abstract}

Key words: Fahr's disease, basal ganglia, calcification.

J Dhaka Med Coll. 2011; 20(1): 86-88.

\section{Introduction:}

Fahr's disease is a rare degenerative neurological disorder characterized by the presence of abnormal calcium deposits and associated cell loss in the areas of brain that control movement, including basal ganglia and cerebral cortex $^{1-4}$. The condition is often referred to as idiopathic basal ganglia calciûcation because there is no apparent explanation for such calciûcation. The pathogenesis is not known, but may be secondary to impairment of the blood brain barrier or to a neuronal calcium phosphorus metabolism disorder ${ }^{1}$. According to reports in medical literature, Fahr's disease is often familial ${ }^{5,6}$. It is believed to have autosomal dominant inheritance but a few cases have been reported to have autosomal recessive inheritance and even some sporadic cases have been reported in literature ${ }^{7}$. The association between the abnormal phenotypes and abnormal genes remain unclear despite the recent mapping to chromosome $14 \mathrm{q}$ of a susceptible locus for Fahr's disease.

\section{Case report:}

A 2 year-9 month old male child, completely immunized only issue of non-consanguineous parents presented with delayed developmental milestones. He achieved his neck control at 4 months of age. She attained his sitting with support at the age of 2 years (Fig. 1) and could not stand or walk. The child also developed

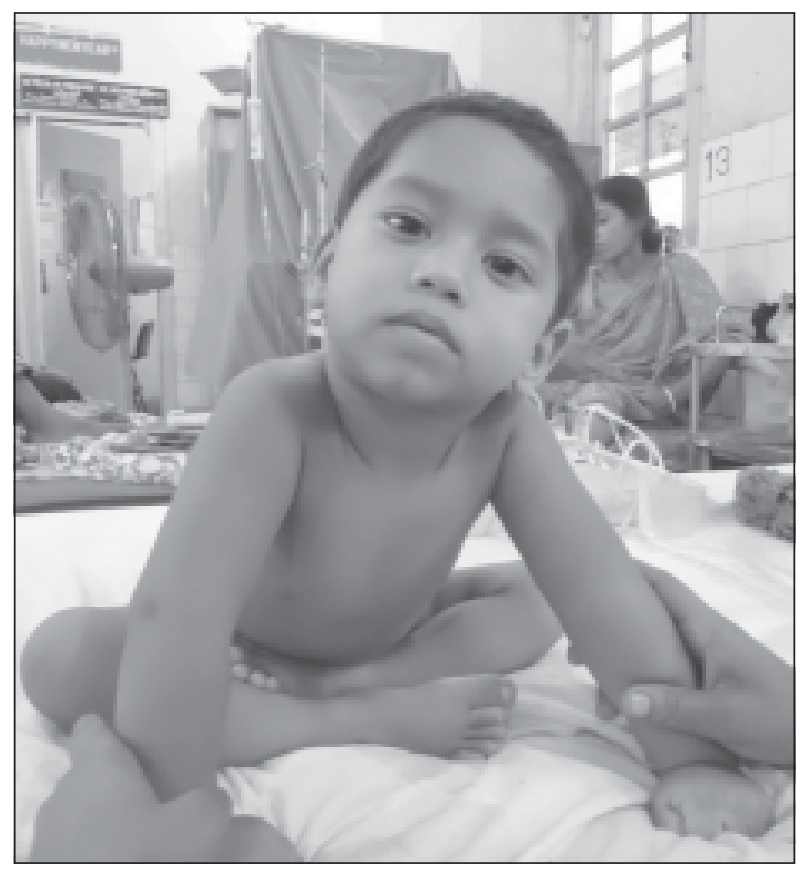

Fig. 1: A 2 year 9 month child can sit only with support.

1. Dr. AKM Matiur Rahman, Assistant Professor of Paediatrics, Dhaka Medical College, Dhaka.

2. Dr. Rashida Sadia Begum, Assistant Professor of Physiology, Dhaka Medical College, Dhaka.

3. Dr. Mohammad Zaid Hossain, Assistant Professor of Medicine, Dhaka Medical College, Dhaka.

4. Dr. Md. Ramzan Ali, Indoor Medical Officer, Department of Paediatrics, Dhaka Medical College Hospital, Dhaka.

5. Dr. Mowshumi Rahman, Assistant Registrar, Department of Pediatric Nephrology, Dhaka Medical College Hospital, Dhaka.

Correspondence: Dr. A.K.M. Matiur Rahman, Assistant Professor of Paediatrics, Dhaka Medical College, Dhaka. Cell Phone: +8801819242859, Email: pediatrics@yahoo.com 
seizure for one episode which involved in face and upper limb with no neurological deficit. $\mathrm{He}$ uttered only Ma..., Ba..., Da..., etc. There was no family history of any similar illness or any other neurological disorder. His birth history was uneventful. General examination was normal. CNS examination showed increased tone in all four limbs especially lower limbs. Deep tendon reflexes were exaggerated with bilateral Babinski sign. Sensory function and cranial nerves were intact. Fundus examination was normal. All the other systems were normal on examination. Plain radiographs of the skull demonstrated irregular calcifications in bilateral fronto-parietal region of skull. A contrast enhanced CT scan of brain was done which revealed bilaterally symmetrical non-enhancing hyperdense lesions of calcification, involving basal ganglia, thalami and subcortical white matter (Fig.2). Blood chemistry revealed normal serum levels of calcium, phosphorus and alkaline phosphatase. The CT scan findings when correlated with clinical history and normal blood chemistry were suggestive of Fahr's disease. Only the conservative treatment was advised to avoid respiratory tract infection. Physical and speech therapy was advised

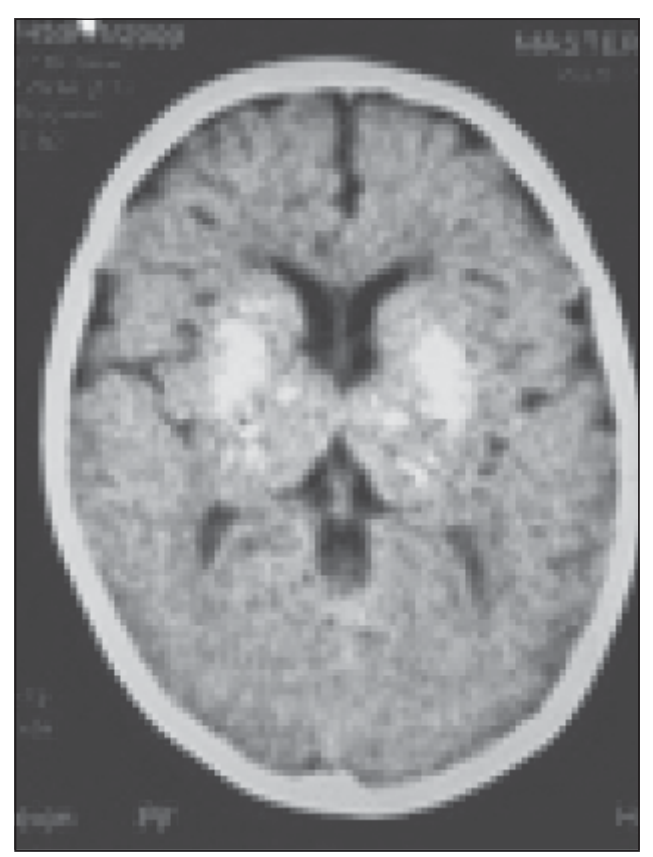

Fig.-2: CT scan image showing multiple symmetric calcifications in basal ganglia, thalamus, subcortical white matter.

\section{Discussion:}

Fahr's disease or familial idiopathic basal ganglia calcification is characterized by bilateral basal ganglia calcification. The most common site of calcification is the globus pallidus. However additional areas of calcification are putamen, caudate nucleus, internal capsule, dentate nucleus, thalamus, cerebellum and cerebral white matter. The calcium deposits occur in the extracellular and extravascular space often surrounding the capillaries $^{8,9}$. It is not clear whether the calcification in Fahr's disease is a metastatic deposition, secondary to local disruption of blood brain barrier, or is due to disorder of neuronal calcium metabolism. The clinical evolution is that of a degenerative disorder, rather than a developmental disorder. The core clinical features are dysarthria, extrapyramidal signs, ataxia, and progressive deterioration of mentality, loss of motor accomplishments, symmetrical spastic paralysis, athetosis, and eye impairment due to optic atrophy. Radiological diagnosis could be the starting point in guiding the clinician to the possibility of Fahr's disease ${ }^{10}$. Genetic heterogeneity and an anticipatory effect have been observed.

Fahr's disease, however, needs to be distinguished from Fahr's syndrome in which basal ganglia calcification is secondary to some other disorder, such as hypoparathyroidism. Basal ganglia calcification may also be seen in various other conditions like CMV infection, neurocysticercosis, toxoplasmosis, neurobrucellosis, tuberculosis, HIV infection, astrocytomas, calcified infarct, pseudohypoparathyroidism, hyperparathyroidism lead intoxication, hypervitaminosis D, mitochondrial encephalopathies, and leukodystrophic diseases ${ }^{1}$.

There is neither a cure for Fahr's Disease, nor a standard course of treatment. Case reports have suggested that haloperidol or lithium carbonate may help in patients with psychotic symptoms. The prognosis is variable and hard to predict.

\section{Conclusion:}

In our case, the child presented with developmental delay and seizure. All the 
common causes of Fahr's syndrome were excluded and CT scan showed classical symmetrical basal ganglia involvement. This rare case of idiopathic Fahr's disease in a child, which has never been reported in Bangladeshi literature, has been brought out to highlight this unusual condition and its differentiation from the commoner Fahr's syndrome. Patients diagnosed with idiopathic Fahr's syndrome should be monitored, and regular neuropsychiatric evaluation performed.

\section{References:}

1. Malik R, Panday VK, Naik D. Fahr's disease. A rare neurodegenerative disorder. Int $\mathrm{J}$ Radiol Image 2004; 14: 383-4.

2. Hasan A, Bajpai G, Varshney N. Fahr's Disease: case report. J Ind Aca Clini Med 2007; 8: 260-1.

3. Harrington MG, Macpherson P, McIntosh WB, et al. The significance of the incidental finding of basal ganglia calcification on computed tomography. J Neurol Neurosurg Psychiat 1981; 44: 1168-70.

4. Smits MG, Gabreels FJM, Thijssen HOM, et al. Progressive idiopathic strio-pallido-dentate calcinosis (Fahr's disease) with autosomal recessive inheritance: report of three siblings. Europ Neurol 1983; 22: 58-64.

5. Billard C, Dulac O, Boulouche J, et al. Encephalopathy with Calcifications of the basalganglia in children: a reappraisal of Fahr's syndrome with respect to 14 new cases. Neuropediatrics 1989; 20: 12-9.

6. Ellie E, Julien J, Ferrer X. Familial idiopathic striopallidodentate calcifications. Neurology 1989; 39: 381-5.

7. Geschwind DH, Loginov M, Stern JM. Identification of a locus on chromosome 14q for idiopathic basal ganglia calcification (Fahr disease). Am J Hum Genet 1999; 65: 764-72.

8. Brodaty H, Mitchell P, Luscombe G, et al. Familial idiopathic basal ganglia calcification (Fahr's disease) without neurological, cognitive and psychiatric symptoms. Am J Hum Genet 2002 ; 110: 8-14.

9. Ang LC, Rozdilsky B, Alport EC, Tchang S. Fahr's disease associated with astrocytic proliferation and astrocytoma. Canada Surg Neurol 1993; 39: 365-9.

10. Fried R. ed. Developmental Neuropathology. New York: Springer-Verlag; 1989. 541-2. 